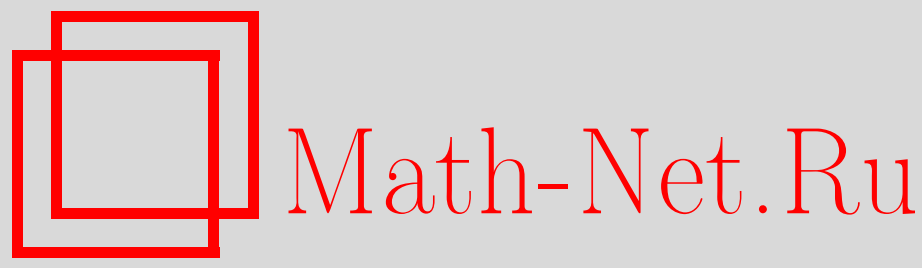

A. Balog, Another Sum-Product Estimate in Finite Fields, Совр. пробл. матем., 2012, выпуск 16, 31-37

DOI: https://doi.org/10.4213/spm31

Использование Общероссийского математического портала Math-Net.Ru подразумевает, что вы прочитали и согласны с пользовательским соглашением http: //www . mathnet.ru/rus/agreement

Параметры загрузки:

IP: 54.172 .240 .79

26 апреля 2023 г., 13:58:01

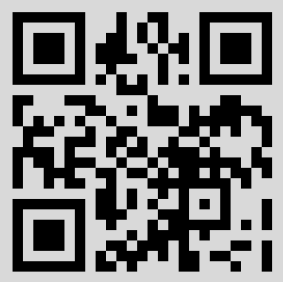




\title{
Another Sum-Product Estimate in Finite Fields
}

\author{
A. Balog \\ Alfréd Rényi Institute of Mathematics, Hungary
}

Dedicated to Professor A. A. Karatsuba on the occasion of his 75 th birthday

\section{Introduction}

Let $A$ and $B$ be subsets of a ring. We denote

$$
A+B=\{a+b: a \in A, b \in B\}, \quad A B=\{a b: a \in A, b \in B\} .
$$

When $A$ is a finite subset of the ring of integers, it is well-known that $|A+A| \geqslant 2|A|-1$ with equality holds for arithmetic progressions, and $|A A| \geqslant 2|A|-1$ with equality holds for geometric progressions. The substantial difference of these two structures led Erdôs and Szemerédi [1] to conjecture that one of $|A+A|$ or $|A A|$ must be big, at least $\gg|A|^{2-\epsilon}$ for any fixed $\epsilon>0$. Although this conjecture is still open, they themselves proved a weaker lower bound, which is gradually improved during the last almost thirty years, the best one is being $\gg|A|^{4 / 3-\epsilon}$, due to Solymosi [2].

Another manifestation of the same phenomenon is that a combination of sets using both addition and multiplication must be big. For example, it is proved in [3] that

$$
|A A+A A+A A+A A| \geqslant \frac{1}{2}|A|^{2} .
$$

Similar questions are also studied in $\mathbb{F}_{q}$, the field of $q$ elements, sometimes for $q$ is a prime. In this situation, being big can be expected as being $\mathbb{F}_{q}$ itself. For example, Glibichuk [4] proved that for $A, B \subset \mathbb{F}_{q},|A||B|>q$ one has $A B+\cdots+A B=\mathbb{F}_{q}$ with 16 terms in the sum. Results become less satisfactory if we change the role of addition and multiplication. Hart, Iosevich and Solymosi [5] proved that

$$
\underbrace{(A-A) \cdots(A-A)}_{d}=\mathbb{F}_{q} \quad \text { if } \quad|A| \gg q^{1 / 2+1 /(2 d)} .
$$

They conjectured that two factors should be enough for $|A| \gg q^{1 / 2+\epsilon}$. Note that without the positive $\epsilon$ this conjecture cannot hold, as is seen from the case of $A=\left\{a \in \mathbb{F}_{q}:|a| \leqslant C q^{1 / 2}\right\}$, where $q$ is a prime, so $\mathbb{F}_{q}$ is the field of residue classes $\bmod q$, and they are identified with $\{a \in \mathbb{Z}:|a|<q / 2\}$. Obviously $A-A=\left\{x \in \mathbb{F}_{q}:|x| \leqslant 2 C q^{1 / 2}\right\}$. It is a classical fact of multiplicative number theory (see Corollary 3 in [6] for the most precise result), that there are at most $\ll C^{2} q / \log ^{c} q$ integers in the form $x y$, where $|x|,|y| \leqslant 2 C q^{1 / 2}$, and $c>0$ is an explicitly computed constant.

15 years earlier Karatsuba [7] and [8] studied sums of Dirichlet characters on additive sets, for example, if $\chi$ is a non principal character $\bmod p$ and $\nu \geqslant 1$ is a fixed integer then he showed

$$
\left|\sum_{a \in A} \sum_{b \in B} \chi(a-b)\right| \ll|A|^{1-1 /(2 \nu)}|B| p^{1 /(4 \nu)}+|A|^{1-1 /(2 \nu)}|B|^{1 / 2} p^{1 /(2 \nu)} .
$$

The author would like to acknowledge the support of the Hungarian National Science Foundation, OTKA K81658.

Special thanks go to Prof. Anirban Mukhopadhyay for stimulating discussions and for the proposal of this problem, to the Institute Mathematical Sciences, Chennai, where the bulk of this work was commenced, and to the anonymous referee for important corrections. 
This estimate is an excellent tool to attack problems like $(A-B) \cdots(A-B)=\mathbb{F}_{q}$, and indeed, a result comparable to (1) can be recovered as its application. Karatsuba based his argument on Weil's bound for Kloosterman's sums, which tool reappears in the work of Hart, Iosevich and Solymosi as well.

In this short note we develop a more elementary method to improve upon (1). This approach merits ideas from Bourgain, Katz, Tao [9], from Glibichuk [4], from Glibichuk, Konyagin [10] and Tao, Vu [11]. It is not entirely elementary, as we are using character sums in proving Lemma 5 below. Actually, this essential part of our argument can be derived from (2), the only reason why we use additive characters instead, is that we get a multiplicative constant of 1 , rather than an implicit constant from (2). We write $\mathbb{F}_{q}^{*}=\mathbb{F}_{q} \backslash\{0\}$. Our results run as follows.

THEOREM 1. Let $A \subset \mathbb{F}_{q}$ with $|A| \geqslant q^{1 / 2+1 / 2^{k}}$, where $k \geqslant 1$. When $A$ is an additive subgroup of $\mathbb{F}_{q}$ then we need in addition that $|A| \geqslant q^{1 / 2}+1$. We have

$$
\underbrace{(A-A)(A-A) \cdots(A-A)}_{2 k+1}=\mathbb{F}_{q} .
$$

Theorem 2. Let $A_{j}, B_{j} \subset \mathbb{F}_{q}$ with $\left|A_{j}\right|\left|B_{j}\right|>8 q$ for $j=1, \ldots, 2 k$, where $k \geqslant 1$, and let $\left|A_{2 k+1}\right|\left|B_{2 k+1}\right|>9 q^{1+1 / 2^{k-1}}$. We have

$$
\left(A_{1}-B_{1}\right)\left(A_{2}-B_{2}\right) \cdots\left(A_{2 k+1}-B_{2 k+1}\right)= \begin{cases}\mathbb{F}_{q} & \text { if } 0 \in \bigcup_{j=1}^{2 k+1}\left(A_{j}-B_{j}\right), \\ \mathbb{F}_{q}^{*} & \text { if } 0 \notin \bigcup_{j=1}^{2 k+1}\left(A_{j}-B_{j}\right) .\end{cases}
$$

In particular, if $|A|>3 q^{1 / 2+1 / 2^{k}}$ then

$$
\underbrace{(A+A)(A+A) \cdots(A+A)}_{2 k+1}= \begin{cases}\mathbb{F}_{q} & \text { if } 0 \in A+A \\ \mathbb{F}_{q}^{*} & \text { if } 0 \notin A+A .\end{cases}
$$

Note that similar results with an even number of factors can be derived by exactly the same principle, but the case of odd factors is somewhat more natural. Actually, the only difference in the results would be a change in the exponent in the size condition. In order to get $2 k$ factors one has to change $1 / 2^{k}$ to $3 / 2^{k+1}$. We leave the details of this minor alteration to the reader. Needless to note that no effort is made to get the best absolute constants in the Theorems.

These results are still far from the conjectural two factors, but stronger than (1) for $k \geqslant 5$. It may be worth to emphasis a partial stage of the proof, (see (6), below), it may have independent interest.

Corollary 1. Let $A \subset \mathbb{F}_{q}$ with $|A|>q^{1 / 2}$, when $|A|$ is an additive subgroup of $\mathbb{F}_{q}$ we need $|A| \geqslant q^{1 / 2}+1$. We have

$$
|\underbrace{(A-A)(A-A) \cdots(A-A)}_{k}|>q^{1-1 / 2^{k}} \text {. }
$$

For example, the conclusion for $k=2$ says $|(A-A)(A-A)|>q^{3 / 4}$. We believe that a stronger lower bound must be true, possibly

Conjecture 1. Let $A \subset \mathbb{F}_{q}$ with $|A|>q^{1 / 2}$, and $\epsilon>0$. We have for $q>q_{0}(\epsilon)$ that

$$
|(A-A)(A-A)| \geqslant|(A-A) A|>q^{1-\epsilon} .
$$

If this conjecture is true, then Lemma 5 below implies that, whenever $|A| \geqslant q^{1 / 2+\epsilon}$ and $q>q_{0}(\epsilon)$, we have

$$
(A-A)(A-A)(A-A)(A-A)(A-A)=\mathbb{F}_{q}
$$




\section{Some Lemmas}

We will need a series of lemmas. Some of them are classical results, but for the sake of completeness we present their proofs, generally it takes a few lines only. Lemma 1 is in the work of Bourgain, Katz and Tao [9], and reappear in the book of Tao and Vu [11]. Lemma 3, the so called Ruzsa's triangle inequality, comes from [12], and an important tool in additive combinatorics. Lemma 4 is folklore, it appears in Vinogradov's book [13], and is the classical approach to estimating a bilinear form.

Lemma 1. Let $A, B \subset \mathbb{F}_{q}$ with $|A||B|>q$. We have

$$
\frac{A-A}{(B-B) \backslash\{0\}}=\mathbb{F}_{q} \text {. }
$$

Proof (see [9]). For any $x \in \mathbb{F}_{q}$ consider the set $A-x B \subset \mathbb{F}_{q}$. Since $|A-x B| \leqslant q<|A||B|$ there are different pairs $\left(a_{1}, b_{1}\right) \neq\left(a_{2}, b_{2}\right)$ such that $a_{1}-x b_{1}=a_{2}-x b_{2}$, that is either $x=$ $\left(a_{1}-a_{2}\right) /\left(b_{1}-b_{2}\right)$, or $a_{1}=a_{2}$ and $x=0$.

Lemma 2. Let $A, B \subset \mathbb{F}_{q}$ with $A \cap B=\varnothing$ and $|A||B| \geqslant 2 q$. We have

$$
\left|\frac{A-B}{A-B}\right| \geqslant \frac{1}{3} q
$$

Proof. For any $x, s \in \mathbb{F}_{q}$ consider the sets $A+x B$, and define the function

$$
R_{x}(s)=\#\{s=a+x b: a \in A, b \in B\} .
$$

Moreover let $\mathcal{X} \subset \mathbb{F}_{q}$ be an arbitrary subset (to be specified later). On one hand we have

$$
\sum_{x \in \mathcal{X}} \sum_{s \in \mathbb{F}_{q}} R_{x}(s)=|\mathcal{X}\|A\| B|
$$

On the other hand an application of the Cauchy-Schwarz inequality imply

$$
\begin{aligned}
|\mathcal{X}|^{2}|A|^{2}|B|^{2} & \leqslant\left(\sum_{x \in \mathcal{X}} \sum_{\substack{s \in \mathbb{F}_{q} \\
R_{x}(s) \neq 0}} 1\right)\left(\sum_{x \in \mathcal{X}} \sum_{s \in \mathbb{F}_{q}}\left(R_{x}(s)\right)^{2}\right) \\
& =\left(\sum_{x \in \mathcal{X}}|A+x B|\right)\left(\sum_{x \in \mathcal{X}} \#\left\{a_{1}+x b_{1}=a_{2}+x b_{2}\right\}\right) \\
& \leqslant\left(\sum_{x \in \mathcal{X}}|A+x B|\right)\left(|\mathcal{X}||A||B|+|A|^{2}|B|^{2}\right),
\end{aligned}
$$

because either $a_{1}=a_{2}, b_{1}=b_{2}$ and than $x \in \mathcal{X}$ is arbitrary, or the quadruple $a_{1} \neq a_{2}, b_{1} \neq b_{2}$ determines at most one $x \in \mathcal{X}$.

By changing the roles of $A$ and $B$ and adding the two inequalities we arrive at

$$
\frac{2|\mathcal{X}|^{2}|A||B|}{|\mathcal{X}|+|A||B|} \leqslant \sum_{x \in \mathcal{X}}(|A+x B|+|x A+B|)
$$

Since $A$ and $B$ are disjoint $a-b \neq 0$ for any $a \in A, b \in B$. If there are different pairs $\left(a_{1}, b_{1}\right) \neq\left(a_{2}, b_{2}\right)$ such that $a_{1}+x b_{1}=x a_{2}+b_{2}$, then $x=\left(a_{1}-b_{2}\right) /\left(a_{2}-b_{1}\right)$. It is time to specify our set $\mathcal{X}$ :

$$
\mathcal{X}=\left\{x \in \mathbb{F}_{q}: x \neq \frac{a_{1}-b_{1}}{a_{2}-b_{2}}\right\}=\left\{x \in \mathbb{F}_{q}:(A+x B) \cap(x A+B)=\varnothing\right\}
$$


For any $x \in \mathcal{X}$ we have $|A+x B|+|x A+B| \leqslant q$, and (3) implies

$$
\frac{2|\mathcal{X}|^{2}|A||B|}{|\mathcal{X}|+|A||B|} \leqslant q|\mathcal{X}|
$$

or with a straightforward calculation from $|A||B| \geqslant 2 q$ we finally conclude

$$
\left|\frac{A-B}{A-B}\right|=q-|\mathcal{X}| \geqslant \frac{|A||B|-q}{2|A||B|-q} q \geqslant \frac{1}{3} q .
$$

LEMma 3. Let $U, V, W$ be finite subsets of an arbitrary group $G$. We have

$$
\left|U V^{-1}\right||W| \leqslant|U W||V W|
$$

Proof (see [12]). We define an injective map from $U V^{-1} \times W$ into $U W \times V W$. To any $r \in U V^{-1}$ we couple a fixed representation $r=u_{r} v_{r}^{-1}$. We map the pair $(r, w) \in U V^{-1} \times W$ to the pair $\left(u_{r} w, v_{r} w\right) \in U W \times V W$. If the pair $(x, y)$ is in the image, then

$$
x y^{-1}=\left(u_{r} w\right)\left(v_{r} w\right)^{-1}=u_{r} w w^{-1} v_{r}^{-1}=r
$$

and so $u_{r}$ and $v_{r}$, as well as $w=v_{r}^{-1} y$ are determined.

Let $\Gamma$ be the group of additive characters of $\mathbb{F}_{q}$ and let $\psi_{0}$ be the principal character. We have the well-known orthogonality relations

$$
\sum_{\psi \in \Gamma} \psi(n)=\left\{\begin{array} { l l } 
{ q } & { \text { if } n = 0 , } \\
{ 0 } & { \text { if } n \neq 0 , }
\end{array} \quad \sum _ { n \in \mathbb { F } _ { q } } \psi ( n ) \left\{\begin{array}{ll}
q & \text { if } \psi=\psi_{0} \\
0, & \text { otherwise }
\end{array}\right.\right.
$$

and the Parseval identity

$$
\sum_{\psi \in \Gamma}\left|\sum_{a \in A} \psi(a)\right|^{2}=q|A|
$$

Lemma 4. Let $A, B \subset \mathbb{F}_{q}$ and $\psi \neq \psi_{0}$. We have

$$
\left|\sum_{a \in A} \sum_{b \in B} \psi(a b)\right| \leqslant(q|A||B|)^{1 / 2}
$$

Proof (see [13]). We start with an application of the Cauchy-Schwarz inequality, and finish with an application of the second relation in (4):

$$
\begin{aligned}
\left|\sum_{a \in A} \sum_{b \in B} \psi(a b)\right|^{2} & \leqslant|A| \sum_{a \in A}\left|\sum_{b \in B} \psi(a b)\right|^{2} \leqslant|A| \sum_{a \in \mathbb{F}_{q}}\left|\sum_{b \in B} \psi(a b)\right|^{2} \\
& =|A| \sum_{a \in \mathbb{F}_{q}} \sum_{b_{1} \in B} \sum_{b_{2} \in B} \psi\left(a b_{1}\right) \overline{\psi\left(a b_{2}\right)}=|A| \sum_{b_{1} \in B} \sum_{b_{2} \in B} \sum_{a \in \mathbb{F}_{q}} \psi\left(a b_{1}-a b_{2}\right)=q|A||B|
\end{aligned}
$$

Lemma 5. Let $A, B, C, D \subset \mathbb{F}_{q}$ with $|A\|B\| C||D| \geqslant q^{3}$. We have

$$
(A+B) C D= \begin{cases}\mathbb{F}_{q} & \text { if } 0 \in(A+B) \cup C \cup D, \\ \mathbb{F}_{q}^{*} & \text { if } 0 \notin(A+B) \cup C \cup D .\end{cases}
$$


Proof. The question of representation 0 is obvious. Let $0 \neq s \in \mathbb{F}_{q}$ be fixed, and we want to express $R(s)$, the number of representations $s=(a+b) c d, a \in A, b \in B, c \in C$, and $d \in D$. Let $C^{*}=\left\{c^{-1}: 0 \neq c \in C\right\}$, and $D^{*}$ is defined analogously. By (4) we have

$$
R(s)=\frac{1}{q} \sum_{\psi \in \Gamma}\left(\sum_{c \in C^{*}} \sum_{d \in D^{*}} \psi(s c d)\right)\left(\sum_{a \in A} \psi(-a)\right)\left(\sum_{b \in B} \psi(-b)\right)
$$

the contribution of the principal character is

$$
=\frac{|A||B|\left|C^{*}\right|\left|D^{*}\right|}{q}
$$

while the contribution of all other characters can be bounded by Lemma 4, by an application of the Cauchy-Schwarz inequality, and finally by (5):

$$
\begin{aligned}
& \leqslant\left(q\left|C^{*}\right|\left|D^{*}\right|\right)^{1 / 2} \frac{1}{q} \sum_{\psi \neq \psi_{0}}\left|\sum_{a \in A} \psi(-a)\right|\left|\sum_{b \in B} \psi(-b)\right| \\
& \leqslant\left(q\left|C^{*}\right|\left|D^{*}\right|\right)^{1 / 2}\left(\frac{1}{q} \sum_{\psi \neq \psi_{0}}\left|\sum_{a \in A} \psi(a)\right|^{2}\right)^{1 / 2}\left(\frac{1}{q} \sum_{\psi \neq \psi_{0}}\left|\sum_{b \in B} \psi(b)\right|^{2}\right)^{1 / 2} \\
& =\left(q\left|C^{*}\right|\left|D^{*}\right|\right)^{1 / 2}\left(|A|-\frac{|A|^{2}}{q}\right)^{1 / 2}\left(|B|-\frac{|B|^{2}}{q}\right)^{1 / 2} .
\end{aligned}
$$

Collecting all these terms we arrive at

$$
R(s) \geqslant \frac{|A||B|\left|C^{*}\right|\left|D^{*}\right|}{q}-\left(q\left|A\|B\| C^{*}\right|\left|D^{*}\right|\right)^{1 / 2}\left(1-\frac{|A|}{q}\right)^{1 / 2}\left(1-\frac{|B|}{q}\right)^{1 / 2}>0
$$

whenever

$$
|A||B|\left|C^{*}\right|\left|D^{*}\right|>q^{3}\left(1-\frac{|A|}{q}\right)\left(1-\frac{|B|}{q}\right) .
$$

If $0 \in C$ then

$$
\left|C^{*}\right|=|C|-1=|C|\left(1-\frac{1}{|C|}\right)>|C|\left(1-\frac{|A|}{q}\right),
$$

if $|A||C|>q$, and similarly with the pair $B, D$, hence the Lemma follows from the conditions $|A||C|>q,|B||D|>q$, and $|A||B||C||D| \geqslant q^{3}$. Note that the first two inequalities follow from the third, because we can trivially assume $|A|,|B|,|C|,|D|<q$.

\section{Proof of the Theorems}

The proof of the two Theorems are basically identical, actually Theorem 1 follows from Theorem 2 up to the constants in the conditions. However we prefer to present an independent treatment, as the proof of Theorem 1 is really short, elegant, and illuminating, while the proof of Theorem 2 follows the same line but needs some unpleasant calculation.

We start the proof of Theorem 1 with putting $U=V=(A-A) \backslash\{0\}$, and $W=V^{\ell}$ for $\ell=1,2, \ldots$ into Lemma 3 , and take $G=\mathbb{F}_{q}^{*}$. Lemma 1 says that asking only $|A|>q^{1 / 2}$, with these choices we have $\left|U V^{-1}\right|=q-1$, hence we conclude

$$
(q-1)\left|V^{\ell}\right| \leqslant\left|V^{\ell+1}\right|^{2}
$$


and with a straightforward induction we get

$$
(q-1)^{2^{\ell}-1}|V| \leqslant\left|V^{\ell+1}\right|^{2^{\ell}} .
$$

Finally using the trivial bounds $q^{1 / 2} \leqslant|A-A|-1=|V|$, which follows from $|A|>q^{1 / 2}$ (or $|A| \geqslant q^{1 / 2}+1$ in case of $A$ is an additive subgroup of $\left.\mathbb{F}_{q}\right)$, and $V^{\ell}=(A-A)^{\ell} \backslash\{0\}$ we have

$$
\left((q-1)^{2^{\ell}-1} q^{1 / 2}\right)^{1 / 2^{\ell}} \leqslant\left|(A-A)^{\ell+1}\right|-1 .
$$

In particular

$$
\left|(A-A)^{k}\right| \geqslant(q-1)^{1-1 / 2^{k}}+1>q^{1-1 / 2^{k}},
$$

which is stated as Corollary 1.

Next putting $B=-A, C=D=(A-A)^{k}$ into Lemma 5 , where $k \geqslant 1$, we get that $(A-A)^{2 k+1}=$ $\mathbb{F}_{q}$ provided that $|A|\left|(A-A)^{k}\right|>q^{3 / 2}$, which is satisfied by (6) if $|A| \geqslant q^{1 / 2+1 / 2^{k}}$. This completes the proof of Theorem 1.

To prove Theorem 2 we have to make some obvious changes. Rather than Lemma 1 we start with Lemma 2 , which requires disjoint sets, not necessarily satisfied by $A_{\ell}$ and $B_{\ell}$. Suppose $\left|A_{\ell}\right|\left|B_{\ell}\right|>8 q$ and split $A_{\ell} \cap B_{\ell}$ to two almost equal halves, say $A_{\ell} \cap B_{\ell}=X \cup Y, X \cap Y=\varnothing$, where either $|X|=|Y|$ or $|X|=|Y|+1$ depending on the parity of $\left|A_{\ell} \cap B_{\ell}\right|$. Let $A_{\ell}^{*}$ and $B_{\ell}^{*}$ be the sets we get by removing $X$ from the bigger of $A_{\ell}$ and $B_{\ell}$, while removing $Y$ from the other. It is obvious that $A_{\ell}^{*} \cap B_{\ell}^{*}=\varnothing, A_{\ell}^{*}-B_{\ell}^{*} \subset A_{\ell}-B_{\ell}$, and $\left|A_{\ell}^{*}\right|\left|B_{\ell}^{*}\right| \geqslant 2 q$. We put $U=V=A_{\ell+1}^{*}-B_{\ell+1}^{*}$ and $W=\left(A_{1}^{*}-B_{1}^{*}\right) \cdots\left(A_{\ell}^{*}-B_{\ell}^{*}\right)$ into Lemma 3. Lemma 2 says that with this choice $\left|U V^{-1}\right| \geqslant q / 3$, hence we conclude

$$
\frac{1}{3} q\left|\left(A_{1}^{*}-B_{1}^{*}\right) \cdots\left(A_{\ell}^{*}-B_{\ell}^{*}\right)\right| \leqslant\left|\left(A_{1}^{*}-B_{1}^{*}\right) \cdots\left(A_{\ell+1}^{*}-B_{\ell+1}^{*}\right)\right|^{2} .
$$

By induction we get

$$
\left(\frac{1}{3} q\right)^{2^{\ell}-1}\left|A_{1}^{*}-B_{1}^{*}\right| \leqslant\left|\left(A_{1}^{*}-B_{1}^{*}\right) \cdots\left(A_{\ell+1}^{*}-B_{\ell+1}^{*}\right)\right|^{2^{\ell}}
$$

Using the trivial bound

$$
\left|A_{1}^{*}-B_{1}^{*}\right| \geqslant \max \left(\left|A_{1}^{*}\right|,\left|B_{1}^{*}\right|\right) \geqslant\left(\left|A_{1}^{*}\right|\left|B_{1}^{*}\right|\right)^{1 / 2} \geqslant(2 q)^{1 / 2}
$$

we arrive at

$$
\left|\left(A_{1}-B_{1}\right) \cdots\left(A_{k}-B_{k}\right)\right|>\left|\left(A_{1}^{*}-B_{1}^{*}\right) \cdots\left(A_{k}^{*}-B_{k}^{*}\right)\right|>\left(\frac{1}{3} q\right)^{1-1 / 2^{k}}
$$

and much the same way we also get

$$
\left|\left(A_{k+1}-B_{k+1}\right) \cdots\left(A_{2 k}-B_{2 k}\right)\right|>\left(\frac{1}{3} q\right)^{1-1 / 2^{k}} .
$$

Next putting $A=A_{2 k+1}, B=-B_{2 k+1}, C=\left(A_{1}-B_{1}\right) \cdots\left(A_{k}-B_{k}\right), D=\left(A_{k+1}-B_{k+1}\right) \cdots$ $\left(A_{2 k}-B_{2 k}\right)$ into Lemma 5 , where $k \geqslant 1$, we get the statement of Theorem 2 provided that

$$
\left|A_{2 k+1}\right|\left|B_{2 k+1}\right|\left|\left(A_{1}-B_{1}\right) \cdots\left(A_{k}-B_{k}\right)\right|\left|\left(A_{k+1}-B_{k+1}\right) \cdots\left(A_{2 k}-B_{2 k}\right)\right|>q^{3},
$$

which is satisfied by (7) and (8) if $\left|A_{2 k+1}\right|\left|B_{2 k+1}\right| \geqslant 9 q^{1+1 / 2^{k-1}}$. This completes the proof. 


\section{Bibliography}

[1] P. Erdős, E. Szemerédi, "On sums and products of integers", Studies in Pure Mathematics, Birkhäuser, Basel, 1983, 213-218.

[2] J. Solymosi, "Bounding multiplicative energy by the sumset", Adv. Math., 222:2 (2009), 402-408.

[3] A. Balog, "A note on sum-product estimates", Publ. Math. Debrecen, 79:3-4 (2011), 283-289.

[4] А.А. Глибичук, "Комбинаторные свойства множеств вычетов по простому модулю и задача Эрдёша-Грэхэма", Матем. заметки, 79:3 (2006), 384-395; A. A. Glibichuk, "Combinational properties of sets of residues modulo a prime and the Erdős-Graham problem", Math. Notes, 79:3 (2006), $356-365$.

[5] D. Hart, A. Iosevich, J. Solymosi, "Sum-product estimates in finite fields via Kloosterman sums", Int. Math. Res. Not. IMRN, 2007, № 5, Article ID rnm007.

[6] K. Ford, "The distribution of integers with a divisor in a given interval", Ann. of Math. (2), 168:2 (2008), 367-433.

[7] А. А. Карацуба, "Распределение значений характеров Дирихле на аддитивных последовательностях", ДАН СССР, 319:3 (1991), 543-545; А. A. Karatsuba, "The distribution of values of Dirichlet characters on additive sequences", Soviet Math. Dokl., 44:1 (1992), 145-148.

[8] А. А. Карацуба, Основы аналитической теории чисел, 2-е изд., перераб. и доп., Наука, М., 1983; A. A. Karatsuba, Basic Analytic Number Theory, Springer-Verlag, Berlin, 1993.

[9] J. Bourgain, N. Katz, T. Tao, "A sum product estimate in finite fields and applications", Geom. Funct. Anal., 14:1 (2004), 27-57.

[10] A. A. Glibichuk, S. V. Konyagin, "Additive properties of product sets in fields of prime order", Additive Combinatorics, CRM Proc. Lecture Notes, 43, Amer. Math. Soc., Providence, RI, 2007, 279-286.

[11] T. Tao, V. Vu, Additive Combinatorics, Cambridge Stud. Adv. Math., 105, Cambridge Univ. Press, Cambridge, 2006.

[12] I. Z. Ruzsa, "On the cardinality of $A+A$ and $A-A$ ", Combinatorics, Vol. II., Colloq. Math. Soc. János Bolyai, 18, North-Holland, Amsterdam, 1978, 933-938.

[13] И. М. Виноградов, Основы теории чисел, 5-е изд., перераб., Гостехиздат, М., 1949; I. M. Vinogradov, Elements of Number Theory, Dover Publ., New York, 1954. 